\title{
Chest X-ray in the emergency department during COVID-19 pandemic descending phase in Italy: correlation with patients' outcome
}

\author{
Chiara Moroni ${ }^{1} \cdot$ Diletta Cozzi $^{1}$ (1) $\cdot$ Marco Albanesi ${ }^{1,2} \cdot$ Edoardo Cavigli $^{1}$ (C) $\cdot$ Alessandra Bindi ${ }^{1} \cdot$ Silvia Luvarà $^{1}$.

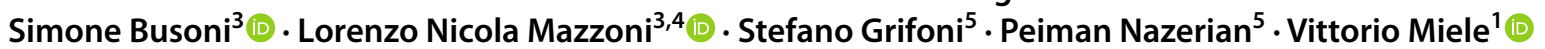

Received: 27 September 2020 / Accepted: 14 December 2020 / Published online: 4 January 2021

(C) Italian Society of Medical Radiology 2021

\begin{abstract}
Purpose The aims of our study are: (1) to estimate admission chest X-ray (CXR) accuracy during the descending phase of pandemic; (2) to identify specific CXR findings strictly associated with COVID-19 infection; and (3) to correlate lung involvement of admission CXR with patients' outcome.

Materials and methods We prospectively evaluated the admission CXR of 327 patients accessed to our institute during the Italian pandemic descending phase (April 2020). For each CXR were searched ground glass opacification (GGO), consolidation (CO), reticular-nodular opacities (RNO), nodules, excavations, pneumothorax, pleural effusion, vascular congestion and cardiac enlargement. For lung alterations was defined the predominance (upper or basal, focal or diffuse, central or peripheric, etc.). Then radiologists assessed whether CXRs were suggestive or not for COVID-19 infection. For COVID-19 patients, a prognostic score was applied and correlated with the patients' outcome.

Results CXR showed $83 \%$ of specificity and $60 \%$ of sensitivity. GGO, CO, RNO and a peripheric, diffuse and basal prevalence showed good correlation with COVID-19 diagnosis. A logistic regression analysis pointed out GGO and a basal or diffuse distribution as independent predictors of COVID-19 diagnosis. The prognostic score showed good correlation with the patients' outcome.

Conclusion In our study, admission CXR showed a fair specificity and a good correlation with patients' outcome. GGO and others CXR findings showed a good correlation with COVID-19 diagnosis; besides GGO a diffuse or bibasal distribution resulted in independent variables highly suggestive for COVID-19 infection thus enabling radiologists to signal to clinicians radiologically suspect patients during the pandemic descending phase.
\end{abstract}

Keywords Chest radiograph · Diagnosis · Emergency department · COVID-19 pneumonia

\section{Abbreviations}

COVID-19 Coronavirus 2019 disease

RT-PCR Reverse transcription polymerase chain reaction

Diletta Cozzi

dilettacozzi@gmail.com

1 Department of Emergency Radiology, Careggi University Hospital, Largo Brambilla 3, 50134 Florence, Italy

2 Department of Clinical and Experimental Medicine, Institute of Diagnostic Imaging 2, University of Sassari, Sassari, Italy

3 Medical Physics Department, Careggi University Hospital, Florence, Italy

4 Medical Physics Unit, AUSL Toscana Centro, Pistoia-Prato, Italy

5 Department of Emergency Medicine, Careggi University Hospital, Florence, Italy

\author{
GGO Ground glass opacity \\ CXR Chest radiography \\ RNO Reticular-nodular opacity \\ $\mathrm{CO} \quad$ Consolidation \\ CT Computed tomography \\ RALE Radiographic Assessment of Lung Edema
}

\section{Introduction}

Since the end of 2019, a global pandemic spread in most countries all over the world. As in many others realities, since the end of February 2020, Italy experienced a first pandemic phase characterized by an exponential increase in the number of affected patients accessing emergency units and, due to government restrictions, a next phase lasting from May to September 2020, characterized by 
a slow progressive reduction of the affected between the ones presenting to hospitals with a clinical suspicion of COVID-19 infection [1].

Imaging played a major role in the management of the infected population, and chest computed tomography (CT) and chest X-ray (CXR) have been extensively used, revealing undisputed utility during the admission workout and the follow-up of hospitalized patients [2-4]. Even if CT imaging is considered the most effective method for the detection of lung abnormalities particularly in the early stage of the disease, portable CXR, in the emergency setting, has played an important role in the identification and follow-up of lung involvement [4-8]. In fact, CXR allowed chest imaging without moving patients from their rooms and so reducing the possibility of a cross-infection among other patients and medical staff and reducing the burden on radiological units, where the recommended times for decontamination of CT rooms have frequently led to a heavy reduction in CT activity $[9,10]$. In our institute, as in many others Italian hospitals, each patient with clinical suspicion of COVID-19 infection underwent a supine CXR in the admission room, as a first-line triage tool in association with reverse transcription polymerase chain reaction (RT-PCR) testing. We evaluated each admission CXR for the presence of a large set of findings, some of which related to COVID-19 pneumonia, as reported in recent literature, and others related to cardio-respiratory non-COVID-19 affections and labeled as suggestive for COVID-19 infection or for an alternative diagnosis [5, 7, 11, 12]. For each COVID-19 patient, each admission CXR has also been scored for the extension of pulmonary findings with a score, previously validated for COVID-19 lung alterations, and all the acquired data were correlated with the patient's final diagnosis and outcome [7].

The aims of our study were to prospectively valuate admission CXR accuracy at the reduction of positive cases between clinically suspects, to identify specific CXR findings strictly associated with COVID-19 final diagnosis and to correlate lung involvement of admission CXR with patients' outcome. Previous studies have focused mainly on description of CXR findings at presentation and on CXR modifications related to the disease time course [5-7, 12]. Other studies were concentrated on the prognostic role of such methodic and have scored radiographic alterations, either with visual scores or using artificial intelligence (AI) technology, generally finding good correlations with patients' outcome $[6,7,13,14]$. Recently, a study by Hare and colleagues has evaluated CXR diagnostic accuracy and detected a very high specificity (100\%) [8]. To our knowledge, however, all previous CXR evaluations were based on the assessment of lung alterations during pandemic peak and were retrospective ones.

\section{Materials and method}

\section{Study design and patients characteristics}

This was an observational monocentric prospective study approved by the Ethical Committee of our referring center (rif. CEAVC 17,104). Informed consent was obtained from study patients. From the 1st to the 30rd of April 2020, we prospectively evaluated all the admission CXR of patients presenting to our institute with a clinical suspicion of COVID-19 infection. According to recent literature, the following characteristics were evaluated: age and gender, fever, cough, dyspnea, reduction of $\mathrm{SpO} 2$, chest pain, myalgias, rhinorrhea, diarrhea, hemoptysis and smell or taste disturbances. All patients underwent a nasopharyngeal swab and a supine CXR while waiting in the isolation ward [11, 15-17]. Results of RT-PCR in respiratory specimens requested in ED were available within $6 \mathrm{~h}$ from the request. We decided not to consider dead patients in relation to the short-term follow-up of our study (one month since the admission date).

\section{CXR examinations}

CXRs were performed in the antero-posterior projection, with patients in the supine position, using portable X-ray units (FDR Go PLUS-Fujifilm, Italy) in the isolation ward. All images were stored in a picture archiving and communication system (PACS, Syngo-Siemens), and a report was provided before moving patients from the admission room. The CXRs were evaluated by two radiologists (a cardiothoracic one and a general one), blinded from any clinical information and, when discordant, the final decision was reached collegially. Case adjudication was dichotomic: COVID-19 infection 'present' or 'absent.' In COVID-19 absent cases, an alternative pre-specified clinical diagnosis was indicated. In each CXR, according to the Fleischer Society glossary of terms and according to previous literature it was searched the presence of ground glass opacity (GGO), consolidation (CO) and reticular-nodular opacities (RNO) [12, 18, 19]. For each CXR was then specified the site of lung alterations as: upper or lower, peripheral or central, focal or diffuse, monolateral or bilateral [12]. Nodules, pleural effusions, lung cavitation, pneumothorax, hilar congestion, peri-bronchial cuffing and cardiac enlargement were also searched and reported when present [18]. Patients with a positive RTPCR in respiratory specimens, comprehensive of bronchoalveolar lavage (BAL) within 5 days from ED presentation, were considered COVID-19 present. In COVID-19 patients only, the extension of the CXR detected findings 
was scored using a severity score validated by Wong and colleagues for COVID-19 lung involvement. It is derived from a previously more complex one developed in order to assess lung edematous involvement in acute respiratory distress syndrome-ARDS (Radiographic Assessment of Lung Edema-RALE) [7, 20]. In this modified RALE score, each lung was classified for the extension of lung involvement from 0 to 4 ( $0=$ no involvement; $1=<25 \%$; $2=25-50 \% ; 3=50-75 \% ; 4=\geq 75 \%$ of involvement), and the scores of both lungs were summed with a maximum value of 8 [7].

\section{Patient diagnosis and outcome}

COVID-19 infection was confirmed by RT-PCR on nasopharyngeal and throat swabs. In our institute, sometimes were needed serial RT-PCR tests before reaching a diagnosis. In very few cases, COVID-19 diagnosis was confirmed with RT-PCR test on BAL. The final diagnosis of patients was that derived from the clinical report for hospitalized patients and from telephonic follow-up for non-hospitalized ones. The outcome of COVID-19 patients was graded considering the following steps: patients discharged (Group 0), patients requiring ordinary hospitalization (Group 1), noninvasive ventilation (Group 2) or intubation (Group 3).

\section{Statistical analysis}

Sensitivity and specificity of CXR examination in the diagnosis of COVID-19 were estimated. The diagnostic accuracy of CXR and the association of each radiological finding with the presence or absence of COVID-19 infection were assessed through Cohen's $K$ test. A forward logistic regression analysis with maximum likelihood method was performed to evaluate the correlations of radiological findings and COVID-19 diagnosis, with sex and age as covariates. Odds ratios and corresponding 95\% confidence intervals (95\% CI) were thus estimated by logistic regression model. The nonparametric test of Kruskal-Wallis was carried out to evaluate the difference in the RALE score between patients with different outcome. Statistical analysis was performed with SPSS (IBM SPSS Statistics for Windows, version 25.0); statistical significance threshold was set with $p=0.05$.

\section{Results}

During the study period, 327 CXRs of patients presenting to our institute with a clinical suspect of COVID-19 infection were evaluated in total. A percentage of 30.5\% (100 patients) resulted COVID-19 positive at nasopharyngeal swab or at BAL RT-PCR tests. The clinical characteristics of the 327 patients and of the 100 infected on presentation
Table 1 Descriptive statistic of total suspects and of COVID-19 population

\begin{tabular}{lll}
\hline Symptoms & Total patients $n=327$ & COVID-19 patients $n=100$ \\
\hline Dyspnea & $168(51 \%)$ & $57(57 \%)$ \\
Fever & $167(51 \%)$ & $75(75 \%)$ \\
Cough & $91(15 \%)$ & $34(34 \%)$ \\
Others & $37(11 \%)$ & $16(16 \%)$ \\
Sex & Males $=161(49 \%)$ & Males $=44(44 \%)$ \\
Age & 69.5 (range 16-101 years) & 71.4 (range 22-100 years) \\
\hline
\end{tabular}

are summarized in Table 1. Note the poor difference in age and sex distribution between the two populations, with a mean age of 71 years for all patients accessing the admission ward and of 69 years for COVID-19 ones. There is also an equal presence of males and females, with only a slight prevalence of the last ones in both cohorts. Likewise, the clinical presentation is very similar with a predominance of fever, dyspnea and cough as presenting symptoms. In Fig. 1 is shown the age stratification of COVID-19 affected patients (Fig. 1). Sensitivity and specificity of CXR examination in the diagnosis of COVID- 19 were $60 \%$ and $83 \%$, respectively (Table 2). CXR accuracy in the diagnosis of COVID-19 was fair (Cohen's $K=0.42$ with a $p$ value $<0.001$ ) (Table 3). It can be observed that findings as GGO, CO, RNO and a peripheral, basal, bilateral and diffuse distribution of lung alterations showed statistically significant Cohen's $K$, indicating an association with COVID-19 diagnosis, although with low $K$ values $(<0.3)$ (Fig. 2, 3). After logistic regression, three radiological findings showed $p$ values $<0.05$ with odds ratio $>1$ : presence of GGO and a basal and diffuse distribution, indicating the three main characteristic that should guide the diagnosis toward COVID-19 infection positivity (Table 4). Nonparametric Kruskal-Wallis test showed statistically significant differences of RALE score between the following groups with different outcomes: $0-1(p=0.014)$, $0-3(p=0.001), 0-2(p=0.03), 1-3(p=0.04)$. These results were obtained despite the large differences in numerosity among groups: Group $0(n=11)$, Group $1(n=85)$, Group 2 $(n=5)$, Group 3 ( $n=5)$ (Fig. 4).

\section{Discussion}

While the high diagnostic accuracy of chest CT in detecting COVID-19 alterations has already been evaluated, with a specificity ranging, according to different studies, from 95 to $99 \%$, and a sensitivity varying from 61 to $98 \%$, to our knowledge, only Hare and colleagues has evaluated CXR specificity [7, 8, 21-24]. They have showed, in their recent work, a CXR specificity of $100 \%$ and a sensitivity of $44 \%$ in diagnosing COVID 19 infection using the British Society 
Fig. 1 Age distribution of patients with COVID-19 pneumonia and positive nasopharyngeal swab

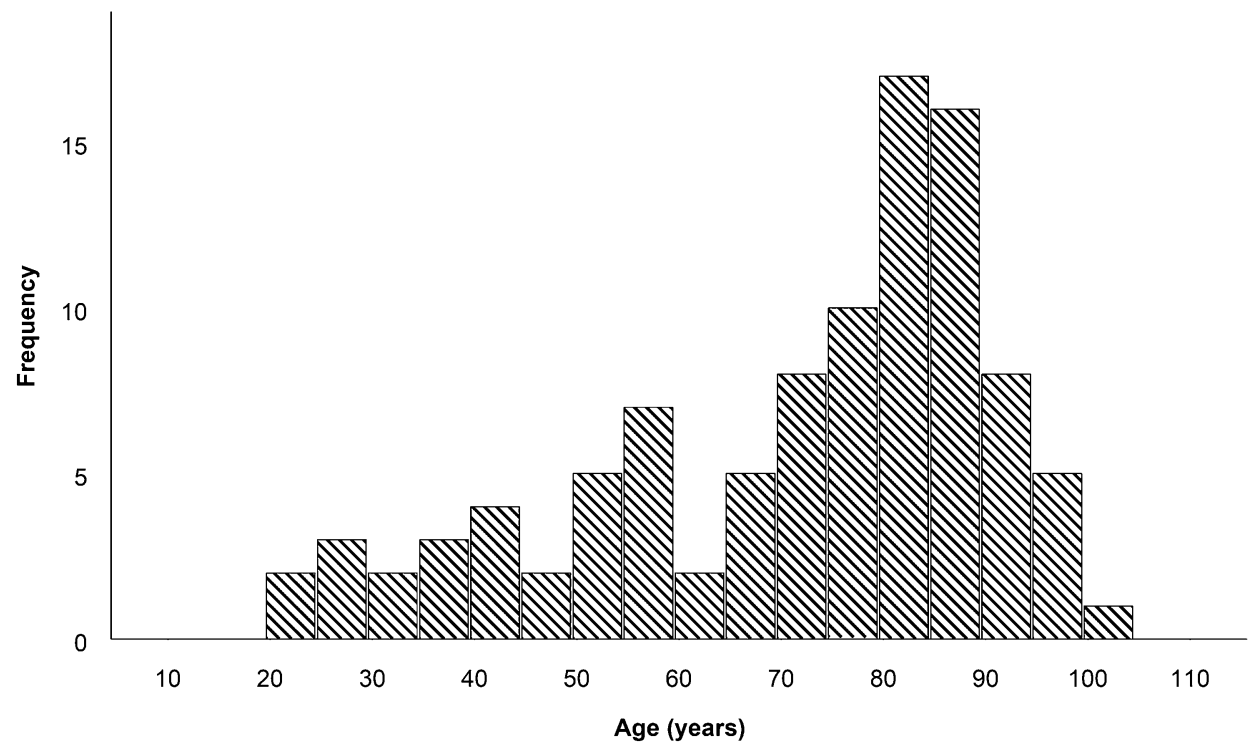

Table 2 CXR examination versus COVID-19 diagnosis contingency table. Reversetranscription polymerase chain reaction (RT-PCR); chest X-ray (CXR)

\begin{tabular}{clll}
\hline \multicolumn{4}{c}{$\begin{array}{l}\text { RT-PCR COVID-19 } \\
\text { diagnosis }\end{array}$} \\
\cline { 2 - 4 } & \multicolumn{1}{c}{ No } & Yes & \multicolumn{1}{c}{ Total } \\
\hline CXR COVID-19 & diagnosis \\
No & 189 & 42 & 231 \\
Yes & 38 & 58 & 96 \\
Total & 227 & 100 & 327 \\
\hline
\end{tabular}

Table 3 Cohen's $K$ for each radiological finding with corresponding $p$ value

\begin{tabular}{lcl}
\hline Radiological finding & Cohen's $K$ & $P$ Value \\
\hline Consolidation (CO) & $\mathbf{0 . 1 8}$ & $\mathbf{0 . 0 0 1}$ \\
Ground glass opacity (GGO) & $\mathbf{0 . 2 8}$ & $<\mathbf{0 . 0 0 1}$ \\
Nodules & -0.28 & 0.26 \\
Reticular-nodular opacities (RNO) & $\mathbf{0 . 1 9}$ & $\mathbf{0 . 0 0 1}$ \\
Peri-bronchial cuffing & -0.70 & 0.17 \\
Hilar congestion & -0.077 & 0.14 \\
Lung cavitation & 0.000 & 1 \\
Pleural effusions & -0.03 & 0.58 \\
Pneumothorax & -0.006 & 0.5 \\
Cardiac enlargement & -0.08 & 0.16 \\
Peripheric distribution & $\mathbf{0 . 2 4}$ & $<\mathbf{0 . 0 0 1}$ \\
Peri-hilar distribution & -0.09 & 0.08 \\
Diffuse distribution & $\mathbf{0 . 2 2}$ & $<\mathbf{0 . 0 0 1}$ \\
Basal distribution & $\mathbf{0 . 1 8}$ & $\mathbf{0 . 0 0 1}$ \\
Superior distribution & 0.01 & 0.67 \\
Monolateral distribution & 0.04 & 0.43 \\
Bilateral distribution & $\mathbf{0 . 1 7}$ & $\mathbf{0 . 0 0 2}$ \\
Left or right distribution & - & 0.32 \\
\hline
\end{tabular}

Statistically significant values are reported in bold font of Thoracic Imaging issued guidelines for the categorization of chest radiographs [25]. Our results show a moderate reduction in CXR specificity (83\%) compared to them. This difference may be related in primis to the different CXR reporting system. In Hare work, patients were categorized as classic/probable COVID-19, indeterminate for COVID19, normal, and non-COVID-19, according to the BSTI guidelines, while we asked the radiologist to assess only the presence or the absence of COVID-19 lung involvement using a set of previously identified radiological findings suggestive for COVID-19 infection, without an "indeterminate" or "normal" pattern [7, 12, 18, 25]. Most likely, our radiologists considered the "indeterminate" and "normal" CXRs as non-COVID-19 ones. In addition, our work is a prospective one, without a pre-selection of patients and may reflect more objectively the Italian reality and, maybe, the diagnostic accuracy of the methodic. Finally, our study took place in a period of non-pandemic peak, with a global reduction of the affected among the ones presenting with a clinical suspicion (in our study, COVID-19 patients were the $30 \%$ of the suspected ones), thereby affecting CXR predictive values in diagnosing COVID-19 patients. All these factors may concur in influencing the evaluation of the methodic diagnostic accuracy. In our opinion, a specificity of $83 \%$, with a negative predictive value of $81 \%$, although not high as those reported by Hare and not high as those reported for CT scans, reveal quite a good ability of CXR to rule out others cardiopulmonary affections mimicking COVID-19 at clinical presentation (Fig. 5) [8, 21, 22]. Our correlation of CXR findings with the final diagnosis of COVID-19 infection identified GGO, a peripheral and a diffuse distribution of lung alterations, as the ones highly related to COVID-19 final diagnosis $(P$ values $<0.001)$. This result is concordant 

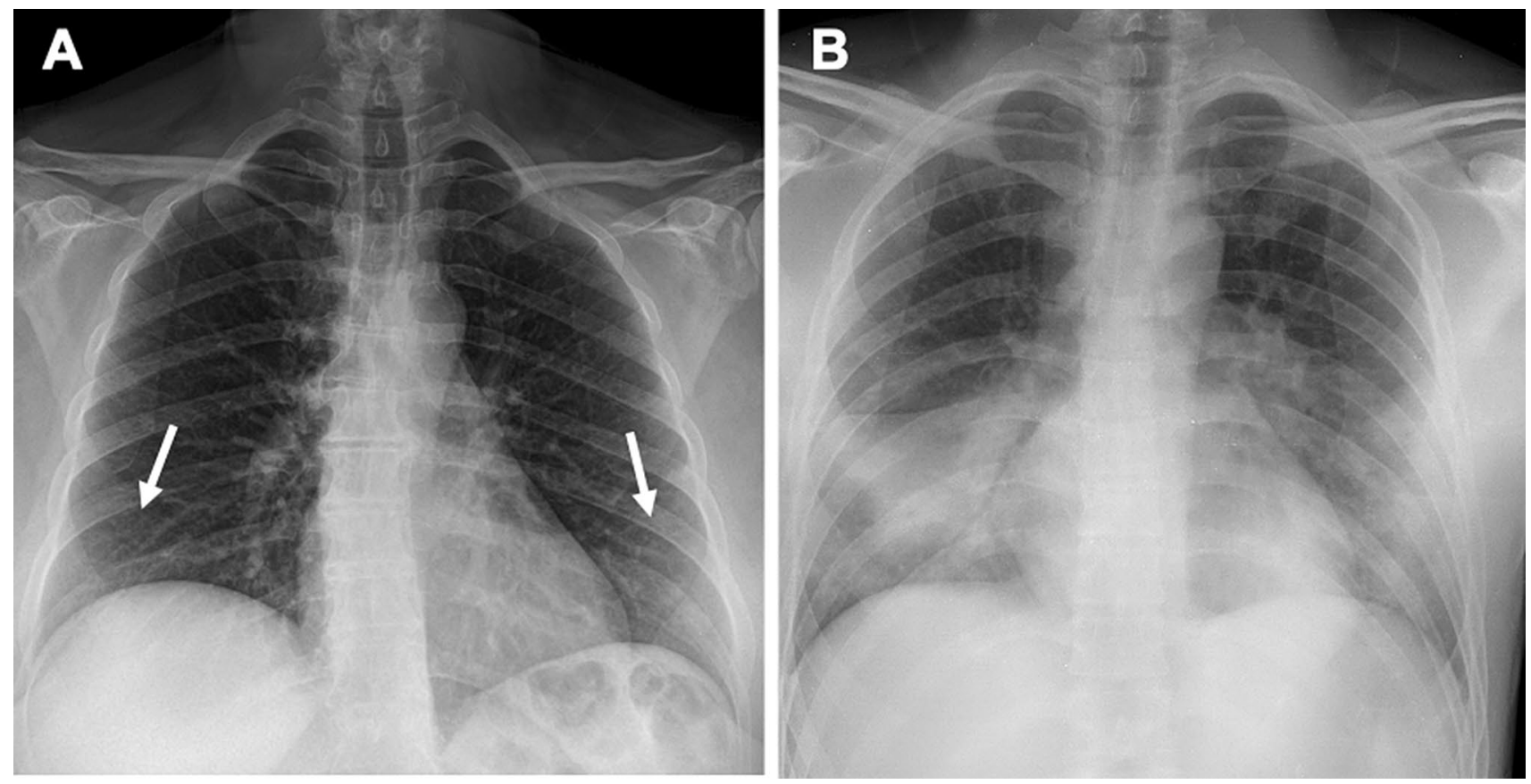

Fig. 2 Ground glass opacities in COVID-19 pneumonia. Figure a shows an initial interstitial thickening in both lower pulmonary lobes (arrows). In figure b, a young male patient with a diffuse basal and bilateral GGO involvement
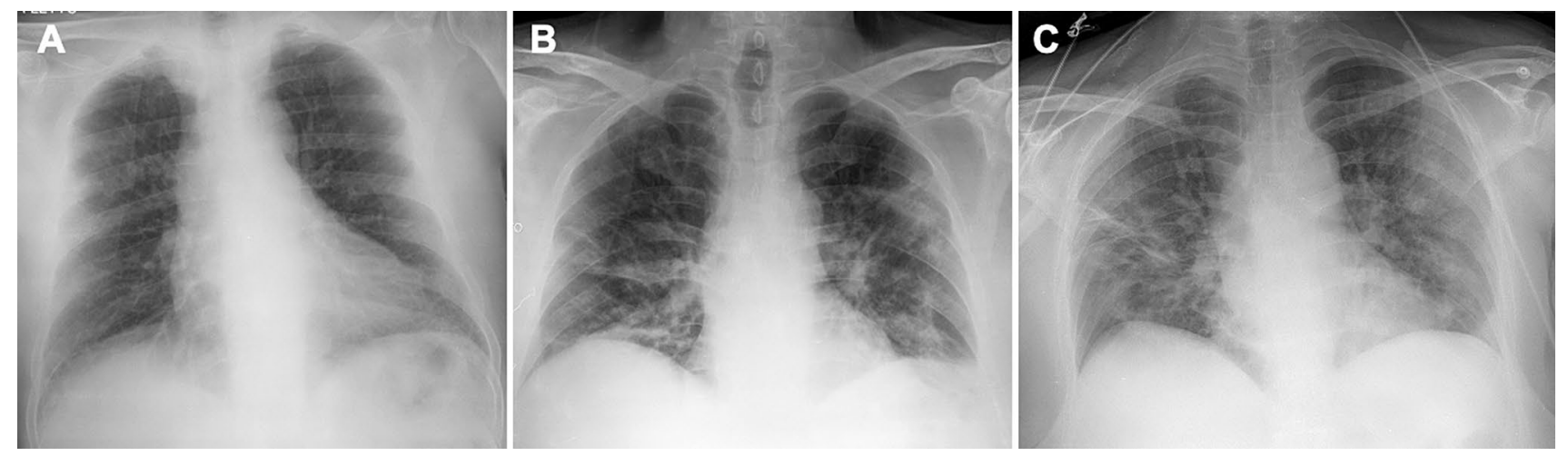

Fig. 3 Diffuse lung involvement in COVID-19 pneumonia. Figure a shows a reticular-nodular pattern in both basal and subpleural parenchyma. Figures in $\mathbf{b}$ and $\mathbf{c}$ demonstrate diffuse lung involvement with bilateral consolidations and thickening of peri-bronchovascular interstitium

Table 4 Logistic regression results. Odds ratio with 95\% confidence interval and $p$ values of radiological findings showing significative association with COVID-19 diagnosis and odds ratio $\geq 1$ after logistic regression. Ground glass opacities (GGO), distribution (D), confidence interval (CI)

\begin{tabular}{llll}
\hline Radiological findings & $P$ value & Odds ratio & $95 \%$ CI \\
\hline GGO & 0.011 & 2.3 & $1.2-4.3$ \\
Diffuse D & 0.002 & 3.11 & $1.5-6.5$ \\
Basal D & 0.02 & 2.1 & $1.1-3.9$ \\
Constant & 0.000 & 0.22 & \\
\hline
\end{tabular}

with previous ones and confirms the necessity for the radiologist to take special attention in these findings as suspicious of COVID-19 lung involvement [8, 12]. Furthermore, a logistic regression carried out between a huge number of variables including all radiographic findings (correlated or not correlated with COVID-19 diagnosis) plus patients' sex and age identified GGO and a diffuse bibasal prevalence of lung alterations as the three independent variables as predictors of COVID-19 diagnosis. These results are significant as they enable radiologists to identify, with enough confidence, 
Fig. 4 Box and Whisker plot of RALE score estimated in each group by outcome: patients discharged (Group 0), patients requiring ordinary hospitalization (1), noninvasive ventilation (2) or intubation (3). RALE score showed a statistical correlation with the patients' outcome confirming the prognostic value of CXR scores of COVID-19 involvement at the diagnosis

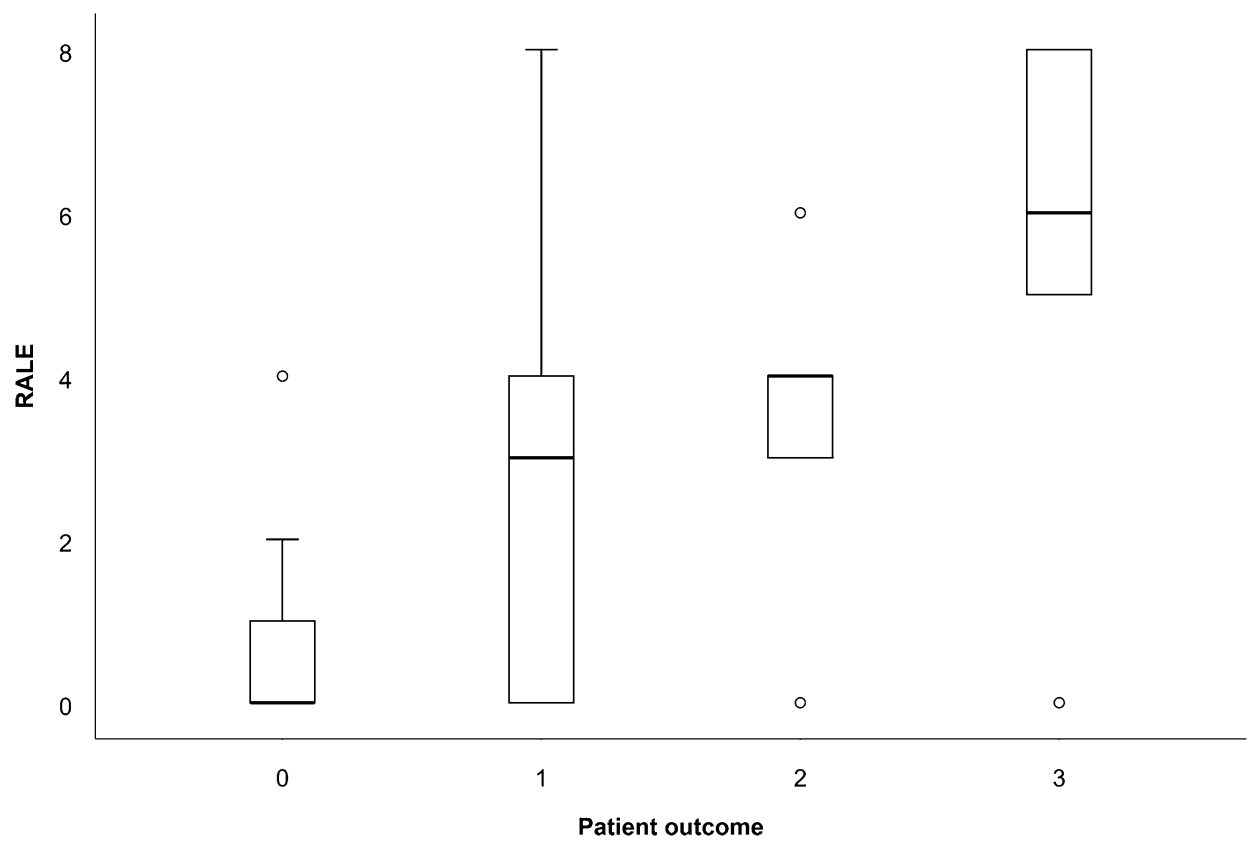

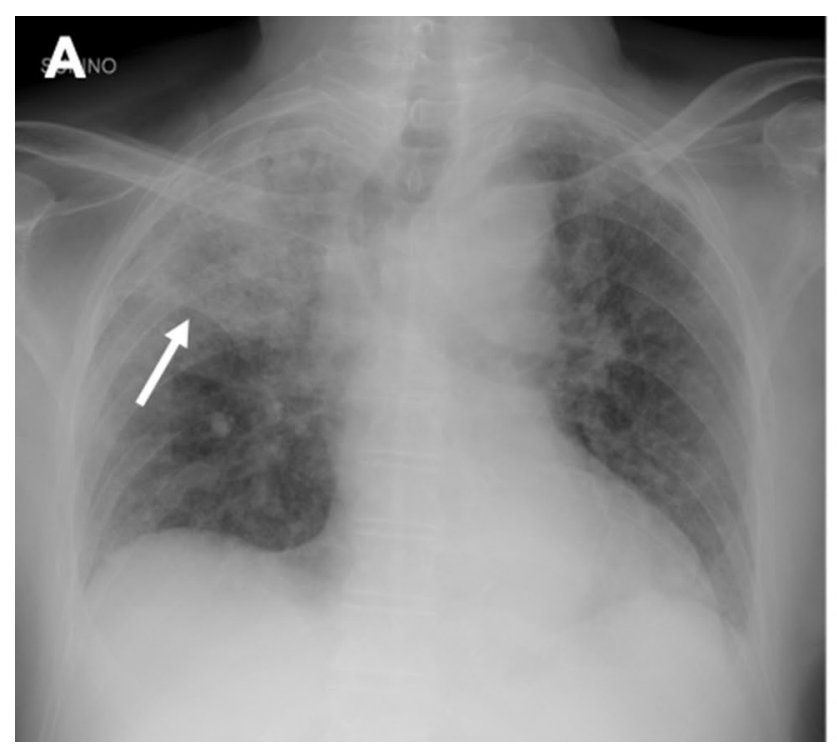

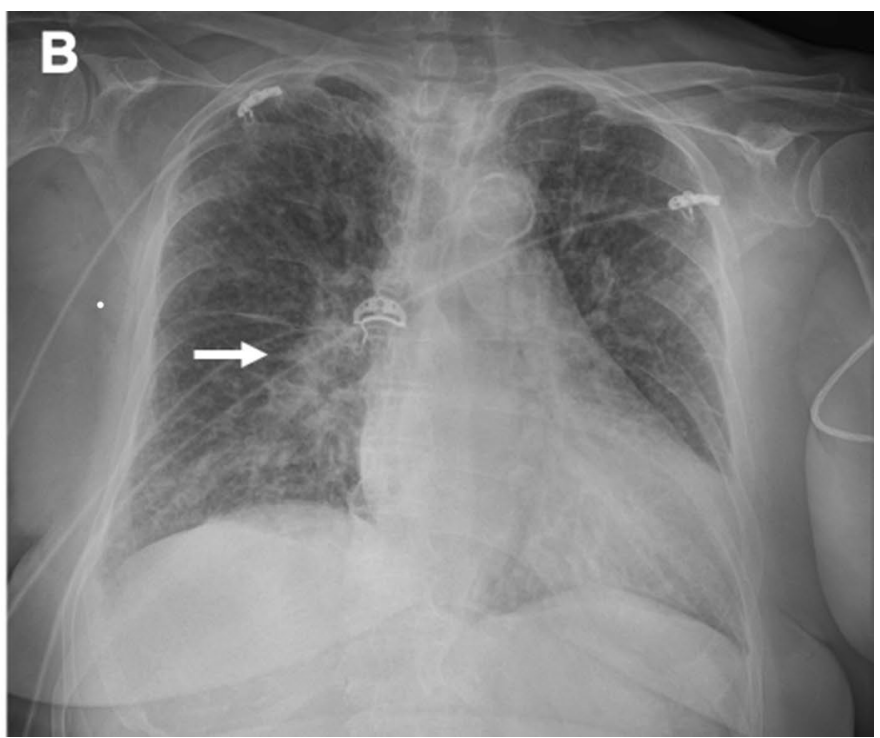

Fig. 5 Alternative diagnosis. In figure a, an upper-right lobar pneumonia (arrow) with diffuse inflammatory lung involvement. In figure b, an 82-year-old woman with cardiac failure and pulmonary edema, hilar congestion (arrow) and cardiomegaly

a subset of radiologically suspected patients among those presenting with similar symptoms in a non-pandemic peak and to ask the clinicians for a different management for the identified ones (such as continue isolation or repeat the negative RT-PCR tests), even in front of a low clinical suspect and of a low prevalence of the infected among the population.

On the other hand, we found a sensitivity of admission CXR of about $60 \%$. This value is similar to that found by Wong et al. (69\%) and by Cozzi et al. (61.1\%) at the pandemic peak and is presumably related to the low capability of CXR to identify subtle lung alterations generally occurring in mild disease or at the beginning of the illness $[7,26]$. This occurring both at the pandemic peak and during the progressive reduction of the affected among the population. In our study, the RALE score showed a good correlation with the patients' outcome confirming the prognostic value of CXR scores of COVID-19 involvement at the diagnosis $[6,7,13,26]$ (Fig. 2). In our experience, $85 \%$ of COVID-19 patients underwent an ordinary hospitalization 
while only a minimum percentage either was dismissed or required respiratory assistance. This evidence reflects the percentage of cases requiring respiratory assistance (approximately 10\%) between the hospitalized ones, similar to that detected during the pandemic peak, and on the other hand reflects Italian policy regarding COVID-19 pandemic [27-29]. In fact, by now in Italy, people are managed at home while experiencing a mild symptomatology and are asked to apply for hospital admission only with the worsening of the clinical situation. These circumstances explain the few cases of non-hospitalized COVID-19 patients (11\%) between all the ones admitting to our institute. This behavior may also explain our slightly higher sensitivity (60\%) compared to Hare's one (44\%) as we have a lower percentage of mild cases between admitted patients, thus possibly lacking a sensible percentage of false negative CXR. In our study, sex and age were not predictors of COVID-19 infection. In fact, sex and age distribution in affected and non-affected populations are similar with an average of 70 years approximately (Table 1). In the affected population, we found a peak of incidence in patients aging between 80 and 85 years. So, although age did not result as a predictor factor for COVID19 infection, the age stratification of our COVID-19 population reflects those previously described [30].

Our study has several limitations. First, we lack interobserver agreement in the diagnostic evaluation and in the prognostic scoring of the CXR (all decisions were reached collegially) while this evaluation has been previously carried out both for diagnostic and prognostic assessment with acceptable results $[6-8,13,25]$. Also, we evaluated only admission CXR and we lack follow-up imaging. This is the main limit of our study as it has been previously proved that CXR findings differ according to different time interval from the clinical onset and that radiological findings strongly modify along with the time course of the disease [7-19]. Furthermore, we have a limited period of follow-up with necessarily an incomplete picture of patients' outcome. This may be a bias affecting the results of the correlation between the modified RALE score and patients' outcome.

However, to our knowledge this is the first prospective study aimed to define CXR diagnostic accuracy, and it is the first study performed during the descending phase of COVID-19 pandemic. This work demonstrates that, although not sensible and specific as chest CT, CXR examination, a convenient fast and bed-side methodic, correlates with patients' outcome and shows a good specificity, thus enabling the radiologist to rule out most of others cardiopulmonary affections. We have also identified three findings (presence of GGO and diffuse and basal lung alterations) which allows radiologists to point out to the clinician highly suspicious patients even in front of a low clinical suspect.

Acknowledgements None.
Author contributions $\mathrm{CM}, \mathrm{DC}, \mathrm{MA}, \mathrm{EC}, \mathrm{AB}, \mathrm{SL}$ helped in radiological data evaluation, manuscript editing; SB NLM contributed to statistical analysis; SG and PN were involved in patients collection; VM helped in final approving.

Funding Authors did not receive support from any organization for the submitted work.

\section{Compliance with ethical standards}

Conflict of interest The authors declare that they have no conflict of interest related to the publication of this article.

Ethical approval All procedures in studies involving human participants were in accordance with the ethical standards of the institutional and/or national research committee and with the 1964 Helsinki Declaration and its later amendments or comparable ethical standards. This study was approved by the Ethics Committee of our University Hospital (rif. CEAVC 17104).

Consent to publish Patients signed informed consent regarding publishing their data and photographs.

Informed consent Informed consent was obtained from all individual participants included in the study. This study was approved by the Ethics Committee of our university hospital (rif. CEAVC 17104). Informed consent was obtained from all individual participants included in the study.

\section{References}

1. Akin L, Gozel MG (2020) Understanding dynamics of pandemics. Turk J Med Sci 50(1):515-519. https://doi.org/10.3906/ sag-2004-133

2. Yang W, Sirajuddin A, Zhang X et al (2020) The role of imaging in 2019 novel coronavirus pneumonia (COVID-19). EurRadiol 15:1-19

3. ACR Recommendations for the use of Chest Radiography and Computed Tomography (CT) for Suspected COVID-19 Infection (2020) American College of Radiology. https://www.acr.org/ Advocacy-and-Economics/ACR-Position-Statements/Recom mendations-for-Chest-Radiography-and-CT-for-SuspectedCOVID19-Infection. Accessed 22 Mar 2020

4. Rubin GD, Ryerson CJ, Haramati LB et al (2020) The role of chest imaging in patient management during the COVID-19 Pandemic: a multinational consensus statement from the Fleischner society. Radiology 296(1):172-180

5. Jacobi A, Chung M, Bernheim A, Eber C (2020) Portable chest $\mathrm{X}$-ray in coronavirus disease-19 (COVID-19): a pictorial review. Clin Imaging 64:35-42

6. Borghesi A, Zigliani A, Golemi S et al (2020) Chest X-ray severity index as a predictor of in-hospital mortality in coronavirus disease 2019: a study of 302 patients from Italy. Int J Infect Dis 8(96):291-293

7. Wong HYF, Lam HYS, Fong AH et al (2019) Frequency and distribution of chest radiographic findings in COVID-19 positive patients. Radiology 27:201160

8. Hare SS, Tavare AN, Dattani V et al (2020) Validation of the British society of thoracic imaging guidelines for COVID-19 chest radiograph reporting. ClinRadiol. https://doi.org/10.1016/j. crad.2020.06.005 
9. Driggin E, Madhavan MV, Bikdeli B et al (2020) Cardiovascular considerations for patients, health care workers, and health systems during the coronavirus disease 2019 (COVID-19) pandemic. J Am CollCardiol 75(18):2352-2371. https://doi.org/10.1016/j. jacc.2020.03.031

10. Kooraki S, Hosseiny M, Myers L et al (2020) Coronavirus (COVID-19) outbreak: what the department of radiology should know. J Am CollRadiol 17(4):447-451

11. Struyf T, Deeks JJ, Dinnes J et al (2020) Signs and symptoms to determine if a patient presenting in primary care or hospital outpatient settings has COVID-19 disease. Cochrane Database Syst Rev 7:CD013665

12. Ng MY, Lee EYP, Yang J et al (2020) Imaging profile of the COVID-19 infection: radiologic findings and literature review. RadiolCardiothorac Imaging 2(1):e200034

13. Toussie D, Voutsinas N, Finkelstein M et al (2020) Clinical and chest radiography features determine patients' outcomes in young and middle age adults with COVID-19. Radiology 14:201754. https://doi.org/10.1148/radiol.2020201754

14. Neri E, Miele V, Coppola F, Grassi R (2020) Use of CT and artificial intelligence in suspected or COVID-19 positive patients: statement of the Italian Society of Medical and Interventional Radiology. Radiol Med 125(5):505-508

15. Huang C, Wang Y, Li X et al (2020) Clinical features of patients infected with 2019 novel coronavirus in Wuhan. China Lancet 395(10223):497-506. https://doi.org/10.1016/S0140 $-6736(20) 30183-5$

16. Pascarella G, Strumia A, Piliego $C$ et al (2020) COVID-19 diagnosis and management: a comprehensive review. J Inter Med. https ://doi.org/10.1111/joim.13091

17. Guan WJ, Ni ZY, Hu Y et al (2020) Clinical characteristics of coronavirus disease 2019 in China. N Engl J Med. https://doi. org/10.1056/NEJMoa2002032

18. Hansell DM, Bankier AA, MacMahon H et al (2008) Fleischner society: glossary of terms for thoracic imaging. Radiology 246(3):697-722. https://doi.org/10.1148/radiol.2462070712

19. Vancheri SG, Savietto G, Ballati F et al (2020) Radiographic findings in 240 patients with COVID-19 pneumonia: time-dependence after the onset of symptoms. EurRadiol 30:1-9

20. Warren MA, Zhao Z, Koyama T et al (2018) Severity scoring of lung edema on the chest radiograph is associated with clinical outcome in ARDS. Thorax. https://doi.org/10.1136/thoraxjnl2017-211280
21. Xu B, Xing Y, Peng J et al (2020) Chest CT for detecting COVID19: a systematic review and meta-analysis of diagnostic accuracy. EurRadiol. https://doi.org/10.1007/s00330-020-06934-2

22. Cozzi D, Bargagli E, Calabrò AG et al (2017) Atypical HRCT manifestations of pulmonary sarcoidosis. Radiol Med 123(3):174-184

23. Xu XW, Wu XX, Jiang XG et al (2020) Clinical findings in a group of patients infected with the 2019 novel coronavirus (SARS-Cov-2) outside of Wuhan, China: retrospective case series. BMJ 368:m606. https://doi.org/10.1136/bmj.m606

24. Grassi R, Cappabianca S, Urraro et al (2020) Chest CT computerized aided quantification of PNEUMONIA lesions in COVID-19 infection: a comparison among three commercial software. Int J Environ Res Public Health 17(18):6914

25. BSTI (2020) Thoracic imaging in COVID-19 infection. Guidance for the Reporting Radiologist British Society of thoracic imaging. https://www.bsti.org.uk/media/resources/files/BSTI_COVID -19_Radiology_Guidance_version_2_16.03.20.pdf. Accessed 13 Apr 2020

26. Cozzi D, Albanesi M, Cavigli E et al (2020) Chest x-ray in new coronavirus disease 2019 (COVID-19) infection: findings and correlation with clinical outcome. Radiol Med. https://doi. org/10.1007/s11547-020-01232-9

27. Cesari M, Montero-Odasso M (2020) COVID-19 and older adults. Lessons learned from the Italian epicenter. Can Geriatr J. 23(1):155-159. https://doi.org/10.5770/cgj.23.445

28. Pianura E, Di Stefano F, Cristofaro M et al (2020) COVID-19: a review of the literature and the experience of INMI LazzaroSpallanzani two months after the epidemic outbreak. J Radiol Rev 7:196-207. https://doi.org/10.23736/S2723-9284.20.00022-4

29. Albano D, Bruno A, Bruno F et al (2020) Impact of coronavirus disease 2019 (COVID-19) emergency in Italian radiologists: a national survey. EurRadiol 30(12):6635-6644

30. Li R, Rivers C, Tan Q et al (2020) The demand for inpatient and ICU beds for COVID-19 in the US: lessons from Chinese cities. Medrxiv. https://doi.org/10.1101/2020.03.09.20033241

Publisher's Note Springer Nature remains neutral with regard to jurisdictional claims in published maps and institutional affiliations. 